\title{
Carta ao editor: os autores brasileiros não citam os autores brasileiros ou não valorizam os trabalhos dos autores brasileiros?
}

\section{Letter to the Editor: Do Brazilian Authors Not Mention Brazilian Authors or Do They Not Value the Works of Brazilian Authors?}

Lúcio Honório de Carvalho Júnior ${ }^{1,2,3]}$

${ }^{1}$ Departamento do Aparelho Locomotor, Faculdade de Medicina da
Universidade Federal de Minas Gerais, Belo Horizonte, MG, Brasil
2 Departamento de Medicina, Pontifícia Universidade Católica de
Minas Gerais, Belo Horizonte, MG, Brasil
${ }^{3}$ Médico Ortopedista, Coordenador do Grupo de Cirurgia do Joelho,
Hospital Madre Teresa de Belo Horizonte, Belo Horizonte, MG, Brasil Rev Bras Ortop 2021;56(5):680.

Foi com interesse que li o artigo intitulado "Retorno ao esporte após osteotomia tibial alta com método de cunha de abertura" dos autores Nicolini et al. ${ }^{1}$ (Rev Bras Ortop 2021;56(3):313-319). Os autores demonstram os resultados de retorno ao esporte de 12 pacientes submetidos a este procedimento após seguimento de 12 meses.

Corroborando e amplificando os achados de Giordano et al. ${ }^{2}$ (Rev Bras Ortop 2021;56(2):154-160), "Os autores brasileiros não citam os autores brasileiros: Nada mudou desde 1994", observamos que trabalho semelhante de minha autoria (De Carvalho Jr et al., "Physical Activity after High Tibial Osteotomy for Treatment of Medial Compartment Knee Osteoarthritis, Rheumatology" [Rheumatology (Sunnyvale) 2016; 6:2] http://dx.doi.org/10.4172/2161-1149.1000200) não foi utilizado e não aparece na referência bibliográfica. Neste trabalho, analisamos o retorno à prática desportiva de 174 pacientes
Endereço para correspondência Lúcio Honório de Carvalho Júnior, MD, PhD, Ortopedia, Hospital Madre Teresa, Av. Raja Gabaglia, 1002, Belo Horizonte, Minas Gerais, 30380-090, Brasil (e-mail: luciohcj@gmail.com).

submetidos a este procedimento, acompanhados por 47,9 meses. Acredito que os achados de Giordano et al. poderiam ser ampliados, acrescentado "mesmo quando os autores brasileiros publicam fora da Revista Brasileira de Ortopedia".

\section{Referências}

1 Nicolini AP, Christiano ES, Abdalla RJ, Cohen M, Carvalho RT. Retorno ao esporte após osteotomia tibial alta com método de cunha de abertura. Rev Bras Ortop 2021;56(03): 313-319

2 Giordano V, Lyra J, Bonadiman JA, Lech O. Os autores brasileiros não citam os autores brasileiros: Nada mudou desde 1994. Rev Bras Ortop 2021;56(02):154-160

3 De Carvalho Júnior LH, Soares LFM, Gonçalves MBJ, et al. Physical Activity after High Tibial Osteotomy for Treatment of Medial Compartment Knee Osteoarthritis. Rheumatology (Sunnyvale) 2016;6:200. Doi: 10.4172/2161-1149.1000200 recebido

10 de Março de 2020

aceito

11 de Fevereiro de 2021
DOI https://doi.org/

$10.1055 / \mathrm{s}-0041-1736427$. ISSN $0102-3616$
(C) 2021. Sociedade Brasileira de Ortopedia e Traumatologia. All rights reserved.

This is an open access article published by Thieme under the terms of the Creative Commons Attribution-NonDerivative-NonCommercial-License, permitting copying and reproduction so long as the original work is given appropriate credit. Contents may not be used for commercial purposes, or adapted, remixed, transformed or built upon. (https://creativecommons.org/ licenses/by-nc-nd/4.0/)

Thieme Revinter Publicações Ltda., Rua do Matoso 170, Rio de Janeiro, RJ, CEP 20270-135, Brazil 